The Astrophysical Journal, 509:256-261, 1998 December 10

(C) 1998. The American Astronomical Society. All rights reserved. Printed in U.S.A.

\title{
A WATER MASER FLARE IN W49N: AMPLIFICATION BY A ROTATING FOREGROUND CLOUD D. A. Boboltz, ${ }^{1,2,3}$ John H. Simonetti, ${ }^{2}$ Brian Dennison, ${ }^{2}$ P. J. Diamond, ${ }^{3}$ AND J. A. Uphoff ${ }^{4}$ Received 1998 March 31; accepted 1998 July 14
}

\begin{abstract}
We monitored the $22 \mathrm{GHz} \mathrm{H}_{2} \mathrm{O}$ maser emission toward W49N from December 1989 through May 1990. During this period we observed an outburst in a component at $-66.25 \mathrm{~km} \mathrm{~s}^{-1}$. The flux density of the flaring component increased by a factor of $\gtrsim 10$ to a maximum of $4020 \mathrm{Jy}$ over a period of 24 days and decreased over the following 34 days to $1400 \mathrm{Jy}$ on the last day of monitoring. During the flux increase the line narrowed from about 1.1 to $0.8 \mathrm{~km} \mathrm{~s}^{-1}$; it subsequently rebroadened to $1.0 \mathrm{~km} \mathrm{~s}^{-1}$. Most interestingly, during the flaring behavior the line center shifted by approximately $0.5 \mathrm{~km} \mathrm{~s}^{-1}$ over the 58 day period. To explain the flare, particularly its shifting line center, we present a model of two interacting maser clouds. In the model, saturated maser radiation produced in a background cloud is amplified by an unsaturated, masing foreground cloud. Motion of the foreground cloud across the line of sight produces a flaring line, accompanied by line narrowing. We demonstrate that the observed flare in W49N may be explained by such a model where a rotating foreground cloud passes in front of a nonrotating background cloud. The differential amplification of the background cloud's radiation produces the observed increase in flux density, line narrowing, and systematic shift in center velocity.
\end{abstract}

Subject headings: ISM: individual (W49) - ISM: molecules — masers — radio lines: ISM

\section{INTRODUCTION}

Variations in interstellar maser intensities are sometimes accompanied by line narrowing and small $\left(\sim 0.5 \mathrm{~km} \mathrm{~s}^{-1}\right)$ systematic shifts in the velocity of the line center (see Rowland \& Cohen 1986 for a summary). Our observations of an $\mathrm{H}_{2} \mathrm{O}$ maser flare in W49N show a combination of flux density inversely correlated with line width and a systematic shift of the line's center velocity. It is commonly argued that velocity shifts $\lesssim 0.5 \mathrm{~km} \mathrm{~s}^{-1}$ are due to changes in the relative strengths of the hyperfine components of the $6_{16}-5_{23}$ $\mathrm{H}_{2} \mathrm{O}$ transition (Sullivan 1971, 1973; Matilla et al. 1985; Rowland \& Cohen 1986). Furthermore, Rowland \& Cohen (1986) argued against a kinematic origin for velocity shifts, citing the lack of shifts $\gtrsim 1 \mathrm{~km} \mathrm{~s}^{-1}$. However, the explanation of velocity shifts through changes in hyperfine components may not be viable. There seems to be no physical mechanism by which individual hyperfine components can be preferentially amplified: because of the small separation between the three strongest hyperfine transitions, neither collisional nor optical pumping can selectively invert one of the hyperfine populations (Litvak 1971). Additionally, although Walker (1984) demonstrated the possible presence of hyperfine structure through a statistical analysis of $\mathrm{H}_{2} \mathrm{O}$ masers in W49N, Gwinn (1994) found no evidence for masing of multiple hyperfine components in an analysis of VLBI observations of W49N.

To explain our observations we consider a maser model consisting of two separate, interacting clouds. To account for the observed line shift we include rotation as well as relative motion in the model. Previously, models of interacting masers separated by distances characteristic of starforming regions were used to explain the extreme brightness temperatures $\left(\gtrsim 10^{15} \mathrm{~K}\right)$ sometimes observed in Galactic $\mathrm{H}_{2} \mathrm{O}$ masers (Deguchi \& Watson 1989; Elitzur, McKee, \&

\footnotetext{
${ }^{1}$ MIT Haystack Observatory, Westford, MA 01886.

2 Virginia Polytechnic Institute and State University, Blacksburg, VA 24061.

${ }^{3}$ National Radio Astronomy Observatory, Socorro, NM 87801.

${ }^{4}$ National Radio Astronomy Observatory, Charlottesville, VA 229032475 .
}

Hollenbach 1991; Kylafis \& Pavlakis 1992). Here we exploit this geometry to explain not only the increase in flux density and line narrowing of the flare, but also the observed frequency shift.

\section{OBSERVATIONS AND DATA REDUCTION}

We monitored the $22235.081 \mathrm{MHz} \mathrm{H}_{2} \mathrm{O}$ emission from $\mathrm{W} 49 \mathrm{~N}$ as part of a larger monitoring program (Simonetti et al. 1993). The monitoring program's observations were made using a $25 \mathrm{~m}$ antenna at either Pie Town, New Mexico, or Kitt Peak, Arizona (both are normally part of the National Radio Astronomy Observatory's Very Long Baseline Array, or VLBA ${ }^{5}$ ). All observations were made in left circular polarization; on-source integration times were 20 minutes. Observations of $\mathrm{W} 49 \mathrm{~N}$ were made from December 1989 through May 1990, at intervals of a few days to about a week. Observing dates covering the outburst (unambiguous detection of the flaring line) are shown in Table 1. The observed signals were recorded at the VLBA sites on VLBI Mk II systems and were subsequently autocorrelated using the NRAO Mk II correlator in autocorrelation mode. These correlation functions were converted into spectra using the NRAO Astronomical Image Processing System (AIPS). The resulting spectra have 288 channels, with a channel width of $0.096 \mathrm{~km} \mathrm{~s}^{-1}$ and an LSR velocity range from -90 to $-63 \mathrm{~km} \mathrm{~s}^{-1}$.

We further reduced the data by first removing a flat baseline from each spectrum. We then computed the integrated flux density over the part of each spectrum not containing the flaring component (i.e., from -72 to $-90 \mathrm{~km} \mathrm{~s}^{-1}$ ). The flux density scale for each spectrum was then adjusted to make this integrated flux density equal to the integrated flux density for a chosen nominal day. We then computed crosscorrelation functions between spectra and used them to correct for any small $\left(\sim 0.08 \mathrm{~km} \mathrm{~s}^{-1}\right)$ velocity shifts relative to the nominal day due to digitization errors. Figure 1 shows final spectra at various stages of the flare. Other than

\footnotetext{
5 The National Radio Astronomy Observatory (NRAO) is a facility of the National Science Foundation operated under cooperative agreement by Associated Universities, Inc.
} 
TABLE 1

DATES AND CORRESPONDING VLBA OBSERVATION STATIONS

\begin{tabular}{cl}
\hline \hline Date & VLBA Station \\
\hline 13 Mar $1990 \ldots \ldots$ & Pie Town, NM \\
15 Mar $1990 \ldots \ldots$ & Pie Town, NM \\
19 Mar $1990 \ldots \ldots$ & Kitt Peak, AZ \\
22 Mar $1990 \ldots \ldots$ & Kitt Peak, AZ \\
26 Mar $1990 \ldots \ldots$ & Kitt Peak, AZ \\
30 Mar $1990 \ldots \ldots$ & Kitt Peak, AZ \\
2 Apr $1990 \ldots \ldots$. & Kitt Peak, AZ \\
6 Apr $1990 \ldots \ldots$. & Kitt Peak, AZ \\
11 Apr $1990 \ldots \ldots$ & Kitt Peak, AZ \\
16 Apr $1990 \ldots \ldots$. & Kitt Peak, AZ \\
23 Apr $1990 \ldots \ldots$. & Pie Town, NM \\
4 May $1990 \ldots \ldots$. & Pie Town, NM \\
7 May $1990 \ldots \ldots$. & Pie Town, NM \\
10 May $1990 \ldots \ldots$ & Pie Town, NM \\
\hline
\end{tabular}

the flaring line, no other spectral features varied dramatically.

We determined three time-dependent properties of the flaring line by fitting a single Gaussian to the line on each monitoring day: the flux density at the peak $\left(F_{v}\right)$, the center velocity of the Gaussian $\left(v_{c}\right)$, and the FWHM $\left(\Delta v_{\text {FWHM }}\right)$. The Gaussian was fitted to the spectrum within a window that was adjusted so that the best $\chi^{2}$ fit was produced with the limitation that the window was at least as wide as the line's FWHM. Figure 2 plots these properties as a function of day number in the monitoring program. The flaring line was not unambiguously detected on days earlier than day 100; the monitoring program ended on day 158 . Figure $2 a$ shows the light curve for the flaring line. The peak flux density rises to $4020 \mathrm{Jy}$ over a period of about 24 days and then falls off to a final value of $1400 \mathrm{Jy}$ on the last day of the 58 day period. Figure $2 b$ shows the systematic shift in the radial velocity of

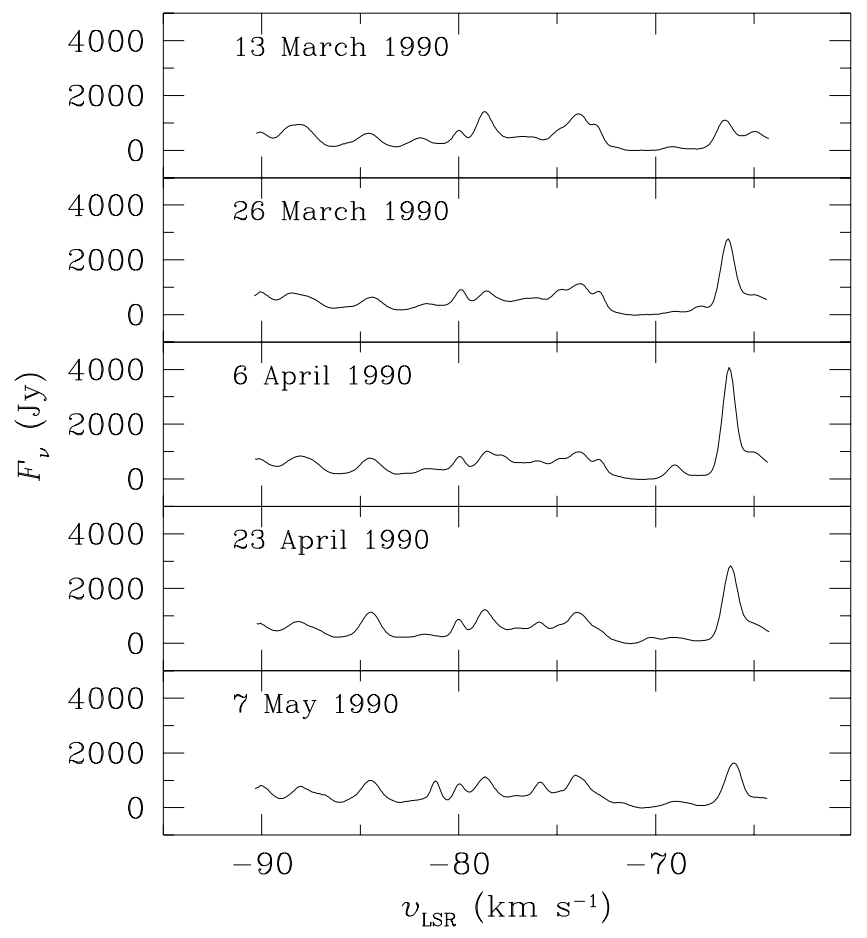

FIG. 1.-Spectra for the $22 \mathrm{GHz} \mathrm{H}_{2} \mathrm{O}$ emission from $\mathrm{W} 49 \mathrm{~N}$ at five epochs. The flaring feature is at $-66.25 \mathrm{~km} \mathrm{~s}^{-1}$. Strong line emission is evident across the entire passband.

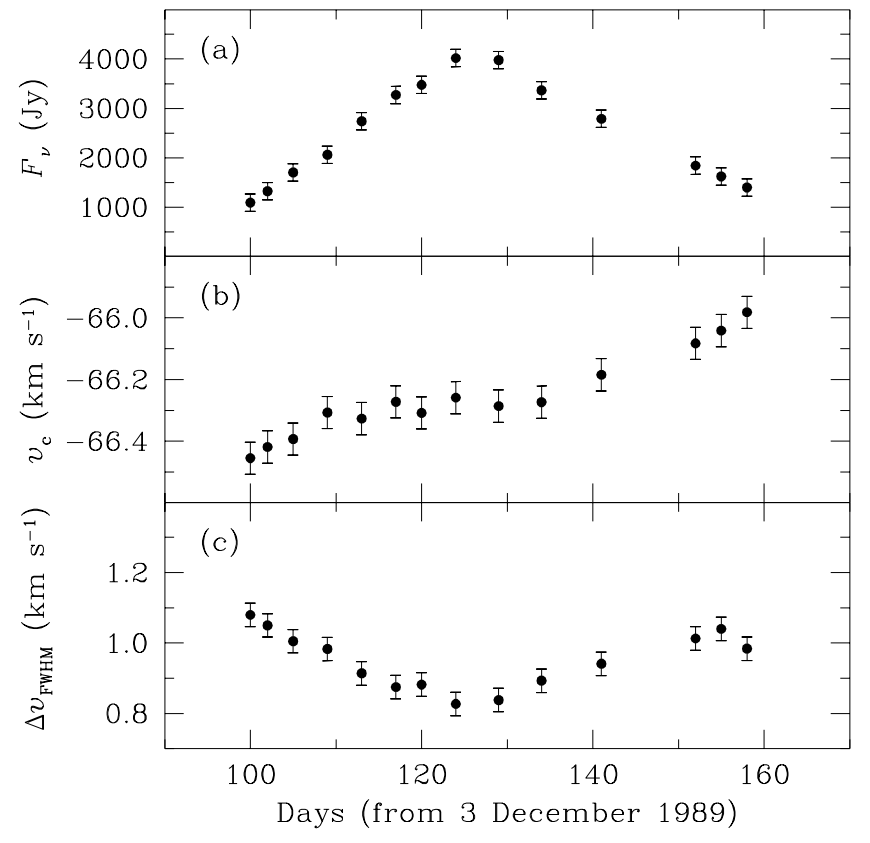

FIG. 2.-Variation of three line parameters obtained from Gaussian fitting of the $-66.25 \mathrm{~km} \mathrm{~s}^{-1} \mathrm{H}_{2} \mathrm{O}$ feature in $\mathrm{W} 49 \mathrm{~N}$. The three parameters, (a) the flux density at line peak $F_{v},(b)$ the velocity of the line center $v_{c}$, and (c) the FWHM line width $\Delta v_{\mathrm{FWHM}}$, are plotted as a function of time in days. Error bars are $1 \sigma$ and were determined as described in the text.

the line center. The total shift is $0.48 \mathrm{~km} \mathrm{~s}^{-1}$ from -66.46 to $-65.98 \mathrm{~km} \mathrm{~s}^{-1}$. Figure $2 c$ shows the narrowing of the line from a maximum line width of $1.08 \mathrm{~km} \mathrm{~s}^{-1}$ to a minimum of $0.83 \mathrm{~km} \mathrm{~s}^{-1}$ at the time of greatest peak flux density. Figures $2 a$ and $2 c$ clearly show that the peak flux density and line width are anticorrelated.

To determine the uncertainties to assign to the measured line properties, we added the best-fit Gaussian for a given day to that day's spectrum at randomly chosen velocities to create several simulated flaring lines. We then fit new Gaussians to these randomly placed lines and found new values for the line properties $F_{v}, v_{c}$, and $\Delta v_{\text {FWHM }}$. We computed the variance for each of the line properties for each monitoring day and used the average variance over the 14 monitoring days to compute the $1 \sigma$ error bars shown in Figure 2. The $1 \sigma$ values for the flux density, center velocity, and line width are $180 \mathrm{Jy}, 0.05 \mathrm{~km} \mathrm{~s}^{-1}$, and $0.08 \mathrm{~km} \mathrm{~s}^{-1}$, respectively.

\section{MODEL}

\subsection{Description}

In this section we construct a model to explain the temporal variation of our measured line properties. We have chosen a simple kinematic model with as few parameters as possible, and we have not attempted to explain all the details present in the data. Rather, our purpose is to demonstrate the viability of a kinematic model for explaining maser flares, particularly flares with a systematic shift in the line-center frequency.

The model employs two separate clouds. Both clouds have population inversions and are assumed to be part of the W49N $\mathrm{H}_{2} \mathrm{O}$ maser complex. As shown in Figure 3, the spherical foreground cloud (cloud 1) passes between the background cloud (cloud 0) and the observer, amplifying the background cloud's radiation and thereby producing 


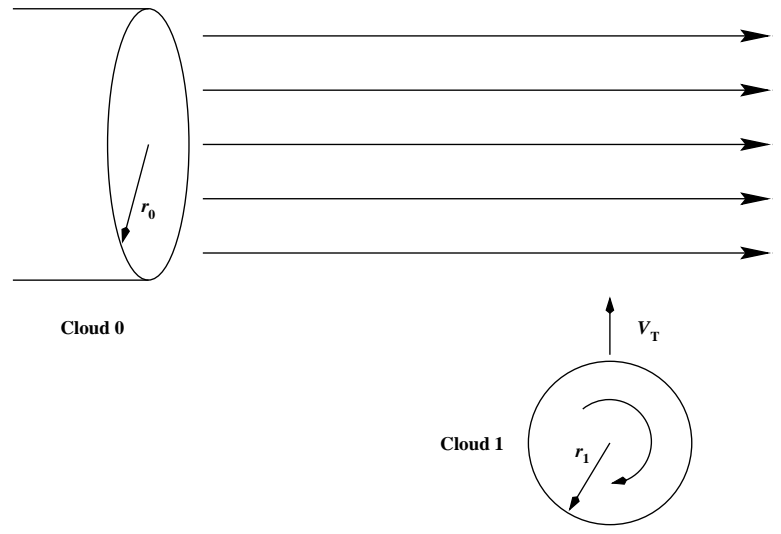

Fig. 3.-Schematic representation of the two interacting masers. The observer is off to the right. The background maser cloud (cloud 0) is, in this figure, represented by a filament, but its exact geometry is not a detail of the model; it is simply assumed to be a uniform source of saturated maser radiation. The spherical foreground cloud (cloud 1) rotates and moves across the line of sight; it amplifies the radiation from the background cloud.

the flare. Assuming a large preflare flux density for the line $\left(\gtrsim 10^{2} \mathrm{Jy}\right.$ ) implies that the background cloud is producing saturated maser radiation; it may be a filament along the line of sight. For the model we simply assume that the background cloud is a Gaussian emission-line source, whose radiation is uniform across its face; in Figure 3 it is represented by a circular source. Thus, the flaring line's behavior is caused by the foreground cloud; its size, motion, and internal parameters determine the results.

To limit the parameters of the model we make a number of simplifying assumptions. Both cloud centers are assumed to have the same line-of-sight velocity (which is zero in the calculations that follow). The only translational motion is the constant-velocity transverse motion of the foreground cloud across the line of sight. At the midpoint of this transverse motion, the centers of the foreground and background clouds are coincident as seen by the observer. We will restrict our presentation to the case where only the foreground cloud rotates; similar results can be obtained with either cloud rotating. The foreground cloud's rotation is strictly rigid body, and the axis of rotation is perpendicular to the line of sight and to the velocity vector of the foreground cloud (i.e., perpendicular to the plane defined by the two clouds and the observer, the plane in which Fig. 3 is drawn). The background cloud's intensity is assumed to be uniform across its face. The internal parameters for the foreground cloud (density, temperature, composition, opacity) are assumed to be uniform. The saturated line produced by the background cloud has a line width equivalent to the width of the Gaussian distribution of its internal thermal and turbulent motions. The foreground cloud is assumed to have the same width for its Gaussian distribution of internal thermal and turbulent motions.

Comparison of the model with observations requires the solution of the equation of radiative transfer. The intensity emergent from the background cloud (cloud 0), at frequency $v$, is $I_{v, 0}$. This radiation is amplified by the foreground cloud. In addition, the foreground cloud (cloud 1) contributes it own unsaturated radiation. Thus, the intensity of a ray emergent from the system is

$$
I(v)=I_{v, 0} e^{\tau_{v, 1}}+S_{v, 1}\left(e^{\tau_{v, 1}}-1\right),
$$

where $\tau_{v, 1}$ is the gain, at frequency $v$, along the path of the ray through the foreground cloud and $S_{v, 1}$ is the source function within the foreground cloud (Elitzur 1992). We have assumed that the background cloud is saturated, producing a very high surface brightness, such that $I_{v, 0} \gg$ $S_{v, 1}$. Therefore, the above equation can be simplified as

$$
I(v) \approx I_{v, 0} e^{\tau_{v, 1}} .
$$

This approximation simplifies the computations, since only rays that originate in the background cloud need to be considered. Henceforth, the frequency $v$ will be replaced with its equivalent line-of-sight velocity $v$.

For an arbitrary ray through the foreground cloud, the gain $\tau_{v, 1}$ depends on the path length through the cloud $\ell_{1}$ and the velocity $v$. The velocity dependence is simple to evaluate since for rigid rotation the line-of-sight velocity is constant along any ray and depends only on $b_{1}$, the distance of closest approach of the ray to the rotation axis. For a Gaussian distribution of thermal and turbulent motions within the cloud, we have

$$
\tau_{v, 1}=\tau_{c, 1}\left(\frac{\ell_{1}}{2 r_{1}}\right) \exp \left\{-\left[\frac{v-v\left(b_{1}\right)}{\Delta v_{D}}\right]^{2}\right\},
$$

where $\tau_{c, 1}$ is the gain along a path through the center of the cloud at zero velocity, $r_{1}$ is the cloud's radius, $\Delta v_{D}$ is the $1 / e$ half-width of the distribution of thermal and turbulent motions within the cloud, and $v\left(b_{1}\right)$ is the mean line-of-sight velocity of material along the ray path due to the solid-body rotation of the cloud.

For a specific position of the foreground cloud, we compute the total output of the system at a given velocity by summing the contributing intensities for a number of uniformly spaced rays passing through the background cloud and (possibly) intersecting the foreground cloud. After repeating the calculation for all relevant velocities, the result is a model spectrum for that position of the foreground cloud. To investigate the basic characteristics of our model the emergent intensities are computed only for rays lying in the plane defined by the line of sight and the transverse velocity vector of the foreground cloud (the plane of Fig. 3). This simplification does not seriously affect the basic results for a spherical foreground cloud as rays far from this central plane do not have as much path length through the foreground cloud and, thus, given the exponential nature of the amplification process, do not contribute much to the total brightness temperature. The full three-dimensional calculation is therefore reduced to a two-dimensional calculation, and the path length of a ray through the foreground cloud is

$$
\ell_{1}=2 r_{1}\left(1-\frac{b_{1}^{2}}{r_{1}^{2}}\right)^{1 / 2}, \text { for }\left|b_{1}\right|<r_{1},
$$

where the value of $b_{1}$ is the vertical displacement of a ray in Figure 3 measured with respect to center of cloud 1 (and so may take on a negative value). Furthermore, the line of sight velocity for the material along a ray in cloud 1 is

$$
v\left(b_{1}\right)=v_{\text {rot }} \frac{b_{1}}{r_{1}},
$$

where $v_{\text {rot }}$ is the rotation speed at the surface of cloud 1 (positive for clockwise rotation as shown in Fig. 3).

To construct a temporal sequence the foreground cloud is moved across the line of sight and a spectrum is obtained 
for each location. By fitting a Gaussian to the line appearing in each spectrum (as was done with the observed spectra) three line parameters are obtained: the amplitude of the line, the center velocity of the line, and the FWHM line width.

The model does not specify the foreground cloud's internal parameters, the physical radius of each cloud, or the distance to Earth, so actual flux densities are not produced. Instead, for comparison with the observed peak flux density values both the model's line amplitudes and the observed peak flux density values (observed line amplitudes) are normalized to unity at the apparent midpoint of the flare. Since the observed maser's size is unknown, this arbitrary scaling of the flux density is appropriate. However, it is worth pointing out that realistic parameters for the model clouds imply that the model results are consistent with the actual observed data, as will be discussed in the next section.

In summary, there are five adjustable parameters in the model: the gain across the center of the foreground cloud at the rest frequency, $\tau_{c, 1}$; the ratio of the cloud radii, $r_{1} / r_{0}\left(r_{1}\right.$ is the radius of the spherical foreground cloud, $r_{0}$ is the radius perpendicular to the line of sight of the background cloud, which may be a filament aligned with the line of sight); the surface rotation velocity of the foreground cloud, $v_{\text {rot }} / \Delta v_{D}$ (expressed in units of the of the $1 / e$ half-width of the internal thermal and turbulent velocity distribution); the transverse velocity of the foreground cloud, $V_{T} /\left(r_{1} /\right.$ day $)$ (expressed in units of foreground cloud radii per day); and $\Delta v_{D}$ (in $\mathrm{km} \mathrm{s}^{-1}$ ), the $1 / e$ half-width of the Gaussian distribution of thermal and turbulent velocities within both clouds, equivalent to the $1 / e$ half-width of the emission line produced by the background cloud. For direct comparison of the model results with the observed data, "days" are used as a unit of time in the model (measured with respect to the apparent midpoint of the flare) and a line-center velocity of zero for the model line is taken to correspond to $-66.25 \mathrm{~km}$ $\mathrm{s}^{-1}$. The scale (in $\mathrm{km} \mathrm{s}^{-1}$ ) of the model's line-center and FWHM results is determined by $\Delta v_{D}$.

\subsection{Model Results and Discussion}

Input parameters for a model computation that reasonably fits the data are shown in Table 2. This model computation (henceforth referred to as the "Model") is not meant to be a "best fit" to the data in a $\chi^{2}$ sense. Rather, we wish to demonstrate the efficacy of kinematic models in accounting for all of the flare's properties. The results of the Model are plotted in Figure 4.

The parameters used in Table 2 are reasonable for typical masers in star-forming regions. The $1 / e$ half-width of 0.85 $\mathrm{km} \mathrm{s}^{-1}$ corresponds to an FWHM of $1.42 \mathrm{~km} \mathrm{~s}^{-1}$ for the saturated maser line emitted by the background cloud, which is a reasonable value (Elitzur, Hollenbach, \& McKee

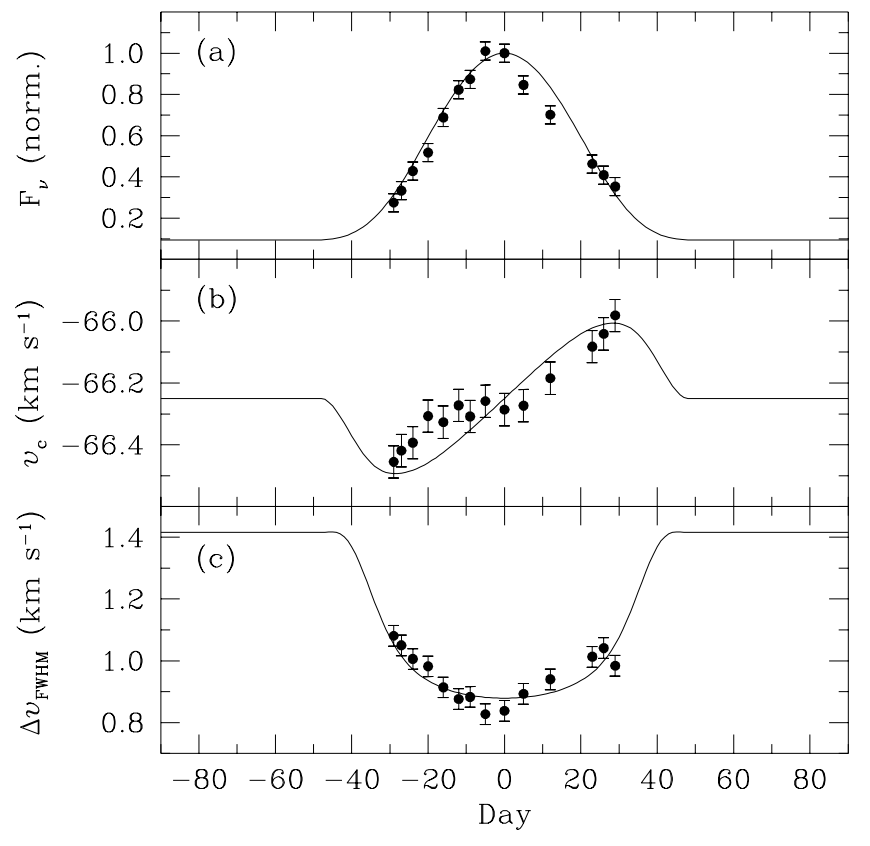

FIG. 4.-A model result with a good fit to the data. Parameters for the model curves are shown in Table 2. The points represent the three observed line parameters as shown in Fig. 1. The observed peak flux density values have been normalized to unity at the midpoint of flare (at day $=0$ here).

1989; Kylafis \& Norman 1991). Taking the background cloud to be a typical $10^{13} \mathrm{~cm}$ in width (dimension across the line of sight), the Model has a foreground cloud that is a factor of 2.75 larger, moving across the line of sight at about $45 \mathrm{~km} \mathrm{~s}^{-1}$. Such relative transverse velocities are found for some components in $\mathrm{W} 49 \mathrm{~N}$ having similar radial velocities and a small angular separation (see Gwinn, Moran, \& Reid 1992). The rotational speed at the surface of the foreground cloud is about $0.8 \mathrm{~km} \mathrm{~s}^{-1}$.

Examining Figure 4 we find that for times far from the midpoint of the flare, where there is no alignment of the clouds, the emergent flux density is small compared with the maximum and is probably undetectable in our experiment. Also, the line's center velocity is equal to the initial -66.25 $\mathrm{km} \mathrm{s}^{-1}$, and the line width is simply that of the background cloud. As the foreground cloud starts to pass in front of the background cloud, the observer sees a rise in peak flux density and an accompanying decrease in the line width, as expected for unsaturated amplification. Furthermore, the line center shifts toward larger negative velocities since the part of the cloud 1 in front of the cloud 0 has a mean velocity toward us (due to the rotation of cloud 1); thus, background cloud emission at negative velocities larger than $-66.25 \mathrm{~km} \mathrm{~s}^{-1}$ is preferentially amplified by the fore-

TABLE 2

PARAMETERS FOR A MODEL COMPUTATION

\begin{tabular}{|c|c|}
\hline Parameter & Value \\
\hline Gain through foreground cloud center $\left(\tau_{c, 1}\right)$ & 2.5 \\
\hline Ratio of cloud radii $\left(r_{1} / r_{0}\right) \ldots$ & 2.75 \\
\hline Surface rotational velocity of foreground cloud $\left(v_{\text {rot }} / \Delta v_{D}\right) \ldots \ldots$ & 0.9 \\
\hline Transverse velocity of foreground cloud $\left[V_{T} /\left(r_{1} /\right.\right.$ day $\left.)\right] \ldots \ldots$ & 0.0285 \\
\hline Thermal/turbulent velocity width $\left(\Delta v_{D}\right) \ldots \ldots \ldots \ldots \ldots \ldots \ldots$ & $0.85 \mathrm{~km} \mathrm{~s}^{-1 \mathrm{a}}$ \\
\hline
\end{tabular}

${ }^{\text {a }} \Delta v_{D}=0.85 \mathrm{~km} \mathrm{~s}^{-1}$ is the $1 / e$ half-width of the Gaussian velocity distribution. The corresponding FWHM is $1.42 \mathrm{~km} \mathrm{~s}^{-1}$. 
ground cloud. As the foreground cloud continues across the line of sight toward the midpoint of its motion, the line center moves toward its unshifted value. Past the midpoint the line shift continues, now toward less negative velocities than the nominal $-66.25 \mathrm{~km} \mathrm{~s}^{-1}$, while the flux density and line width behaviors mirror, in reverse, what took place before the midpoint. These results are a demonstration of the success of this model in explaining the observed characteristics of the flaring line.

As one varies each of the input parameters slightly, the changes in the computational results are not difficult to understand. The transverse velocity $V_{T}$ of the foreground cloud simply determines the timescale of the effects; a larger velocity implies a compressed timescale. Increasing $\tau_{c, 1}$ results in more dramatic (larger amplitude) effects for the increase in the peak of the line, the shift of the line's center velocity, and the decrease in the line width. An increase in the size of the foreground cloud relative to the background cloud $\left(r_{1} / r_{0}\right)$ also increases the amplitude of the effects. This result is due to the relative increase in the amount of material in the foreground cloud with velocities within a specific bin in the spectrum (the radius of the rigidly rotating foreground cloud has increased). Thus, for example, when the foreground cloud is coincident with the background cloud (at the midpoint of the flare), the amount of material in the foreground cloud with velocities nearly equal to the center velocity of the background cloud's line is greater than for a smaller cloud and the line will be more amplified and narrower. Also, when the foreground cloud starts to pass in front of the background cloud, relatively more material in the foreground cloud in a velocity bin on the negative side of $-66.25 \mathrm{~km} \mathrm{~s}^{-1}$ will cause a greater shift in the resulting amplified line. (A side effect of an increase in $r_{1} / r_{0}$ is a compression of the timescale of the effects since the transverse velocity parameter is expressed in units of $r_{1}$ day ${ }^{-1}$.) Increasing the surface rotation velocity $v_{\text {rot }}$ of the foreground cloud increases the amplitude of the shift of the line's center velocity, as one expects (this is the origin of the line shift in this model); there is also a resulting increase in the line width, a reasonable result. Finally, increasing the width of the internal thermal and turbulent cloud velocities distributions, $\Delta v_{D}$, increases the scale of the line shift (as a side effect, since $v_{\text {rot }}$ is in units of $\Delta v_{D}$ ). Additionally, the initial FWHM of the line is larger when $\Delta v_{D}$ is increased, therefore the minimum value of line width reached by the narrowing line is not as low.

In addition to this model, we tried calculations where (1) the background cloud was represented not by a uniform source of radius $r_{0}$, but by a source whose intensity is described by a Gaussian of $1 / e$ radius $r_{0}$, or (2) the foreground cloud was given a Gaussian column density of $1 / e$ radius $r_{1}$. In case 1 the results for the flaring peak flux density, shifting line center, and narrowing line width are essentially identical to our simple model described above. In case 2 the results are essentially identical during the times when observed data points are measurable; the only difference is a more gradual onset of effects at very early times before the flare (and a more gradual approach to the initial values at very late times). These calculations show that our simple model is sufficient for a reasonable exploration of our two-cloud system; the results are not highly dependent on the details of the cloud structures.
It is noteworthy that there may be more detail present in the observed behavior of the line center than is explained by our computed model. In particular, near the midpoint of the flare the line center is nearly constant (and unshifted) for some number of days. Interestingly, for computed models where the foreground cloud is smaller than the background cloud we get precisely this behavior. However, for a small foreground cloud the peak of the flaring line levels off near the midpoint and the line width shows a slow increase then decrease during the time when the line center is nearly constant and unshifted. In all three cases, we are seeing a behavior near the midpoint that can be described as a suppression of the nominal effects of our model (flare, shifted line, narrowed line). We feel that a larger foreground cloud, as in the parameters in Table 2, produces a better overall fit to all the observed data. However, a more complex model than we have attempted here (e.g., a more complex velocity field, not rigid rotation) may be worth exploring.

\section{CONCLUSIONS}

We are able to construct a model of two interacting, masing clouds with simple motions that explains the observed variations in line amplitude, width, and center velocity for a flare in W49N. In our model the background cloud is a simple, uniform source of a saturated maser line. The foreground, unsaturated maser cloud moves across the line of sight as it rigidly rotates, amplifying the emission from the background cloud. The rotation of the foreground cloud produces a systematic shift in the center of the line emergent from the system. The final Model parameters are consistent with values considered typical for $\mathrm{H}_{2} \mathrm{O}$ masers. Our solution is not unique. We were able to achieve reasonable fits to the normalized flux density data with models involving various cloud sizes, for example.

Although previous papers have suggested that systematic shifts in the radial velocity of the line center $\lesssim 0.5 \mathrm{~km} \mathrm{~s}^{-1}$ can be explained by changes in the relative strengths of the hyperfine components of the $22 \mathrm{GHz}$ line, there seems to be no physical mechanism by which this amplification can occur. Our model kinematically generates a shift in line center. The lack of observations of systematic line shifts greater than about $1 \mathrm{~km} \mathrm{~s}^{-1}$ has been used to argue against a kinematic origin and in favor of the hyperfine origin of the shifts. However, in our model the line shift cannot be larger than the value of the line width, approximately, so the absence of large observed line shifts is explained as a natural consequence of the model.

In conclusion, we suggest that flares in $\mathrm{H}_{2} \mathrm{O}$ masers are caused by alignment of masing regions and, most importantly, that accompanying line shifts have a kinematic origin. Our model for demonstrating this is admittedly simplistic. It may be appropriate to consider masing clouds with high aspect ratio and/or more complex velocity fields. The essential ingredients are the alignment of masing regions along the line of sight accompanied by a transverse gradient in the radial velocity field.

The authors wish to thank the referee, Moshe Elitzur, for very useful comments. This research was partially funded by NSF grant AST 93-19670. D. A. B. wishes to acknowledge the support of the NRAO predoctoral fellowship program. 
Deguchi, S., \& Watson, W. D. 1989, ApJ, 340, L17 Elitzur, M. 1992, Astronomical Masers (Dortrecht: Kluwer)

Elitzur, M., Hollenbach, D. J., \& McKee, C. F. 1989, ApJ, 346, 983

Elitzur, M., McKee, C. F., \& Hollenbach, D. J. 1991, ApJ, 367, 333

Gwinn, C. R. 1994, ApJ, 429, 241

Gwinn, C. R., Moran, J. M., \& Reid, M. J. 1992, ApJ, 393, 149

Kylafis, N. D., \& Norman, C. 1991, ApJ, 373, 525

Kylafis, N. D., \& Pavlakis, K. G. 1992, ApJ, 400, 344

Litvak, M. M. 1971, ApJ, 170, 71
REFERENCES

Mattila, K., Holsti, N., Toriseva, M., Anttila, R., \& Malkamaki, L. 1985, A\&A, 145, 192

Rowland, P. R., \& Cohen, R. J. 1986, MNRAS, 220, 233

Simonetti, J. H., Diamond, P. J., Uphoff, J. A., Boboltz, D., \& Dennison, B. 1993, Astrophysical Masers, ed. A. W. Clegg \& G. E. Nedoluha (Berlin: Springer), 311

Sullivan, W. T. III. 1971, ApJ, 166, 321

. 1973, ApJS, 25, 393

Walker, R. C. 1984, ApJ, 280, 618 